\title{
Tumor suppressor function of Bruton tyrosine kinase is independent of its catalytic activity
}

\author{
Sabine Middendorp, A. J. Esther Zijlstra, Rogier Kersseboom, Gemma M. Dingjan, Hassan Jumaa, and Rudolf W. Hendriks
}

\begin{abstract}
During B-cell development in the mouse, Bruton tyrosine kinase (Btk) and the adaptor protein SLP-65 (Src homology 2 [SH2] domain-containing leukocyte protein of $65 \mathrm{kDa}$ ) limit the expansion and promote the differentiation of pre-B cells. Btk is thought to mainly function by phosphorylating phospholipase $C_{\gamma} 2$, which is brought into close proximity of Btk by SLP-65. However, this model was recently challenged by the identification of a role for Btk as a tumor suppressor in the absence of SLP- 65 and by the finding that
\end{abstract}

Btk function is partially independent of its kinase activity. To investigate if enzymatic activity is critical for the tumor suppressor function of Btk, we crossed transgenic mice expressing the kinaseinactive K430R-Btk mutant onto a Btk/ SLP-65 double-deficient background. We found that K430R-Btk expression rescued the severe developmental arrest at the pre-B-cell stage in Btk/SLP-65 doubledeficient mice. Moreover, K430R-Btk could functionally replace wild-type Btk as a tumor suppressor in SLP-65- mice: at 6 months of age, the observed pre-B-cell lymphoma frequencies were approximately 15\% for SLP-65- mice, 44\% for Btk/SLP-65deficient mice, and $14 \%$ for K430R-Btk transgenic mice on the Btk/SLP-65-deficient background. Therefore, we conclude that Btk exerts its tumor suppressor function in pre-B cells as an adaptor protein, independent of its catalytic activity. (Blood. 2005; 105:259-265)

๑ 2005 by The American Society of Hematology

\section{Introduction}

Early in B-cell development, productive V(D)J (variable diversity joining) recombination leads to the synthesis of immunoglobulin (Ig) $\mu$ $\mathrm{H}$ chain protein, which is expressed on the cell surface together with the nonrearranging VpreB and $\lambda 5$ surrogate light-chain (SLC) proteins, and forms the pre-B-cell receptor (pre-BCR; reviewed in Meffre et $\mathrm{al}^{1}$ and Melchers et $\mathrm{al}^{2}$ ). It appears that the pre-BCR has a ligand-independent signal transducing capacity, inducing phosphorylation of the Ig- $\alpha /$ CD79a and Ig- $\beta / C D 79 b$ signaling components and subsequently proliferative expansion and differentiation into large cycling pre-B cells. ${ }^{3,4}$ Pre-BCR signaling involves the formation of a lipid raft-associated signaling module, which is composed of the tyrosine phosphorylated proteins Syk, the Src-family tyrosine kinase Lyn, Src homology 2 (SH2) domain-containing leukocyte protein of $65 \mathrm{kDa}$ (SLP-65; also known as B-cell linker [BLNK] or B-cell adaptor containing $\mathrm{SH} 2$ domain [BASH]), phosphoinositol 3-kinase (PI3K), Bruton tyrosine kinase (Btk), Vav, and phospholipase $\mathrm{C} \gamma 2$ (PLC $\gamma 2){ }^{5-7}$ Activation of PLC $\gamma 2$ by Btk results in the production of the second messengers inositol trisphosphate (IP3) and diacylglycerol (DAG), which activate calcium signaling and protein kinase $\mathrm{C}$ (PKC), respectively. ${ }^{8,9}$ The pre-BCR is transiently expressed on the cell surface, whereby the signaling molecules SLP-65 and Btk are important for the developmental progression of large cycling into small resting pre-B cells in which Ig L chain rearrangement occurs (reviewed in Hendriks and Middendorp ${ }^{10}$ ).

By analogy with the BCR in mature B cells, Btk is thought to be phosphorylated by Syk or Lyn upon recruitment to the pre-B-cell membrane through binding of its pleckstrin homology $(\mathrm{PH})$ domain to the PI3K product phosphatidyl-inositol trisphosphate

From the Department of Immunology, Erasmus MC Rotterdam, Rotterdam, The Netherlands; and Institute for Biology III, Albert-Ludwigs University of Freiburg, and Max Planck Institute for Immunobiology, Freiburg, Germany.

Submitted July 15, 2004; accepted August 14, 2004. Prepublished online as Blood First Edition Paper, August 26, 2004; DOI 10.1182/blood-2004-07-2708.

Supported in part by the Netherlands Organization for Scientific Research (R.W.H.; grant no. 901-07-209).
(PIP3). ${ }^{11,12}$ In this signaling pathway, phosphorylated SLP-65 plays an essential role as it provides docking sites for phosphorylated Btk as well as PLC $\gamma 2$ and thus brings Btk in close proximity with PLC $\gamma 2 .{ }^{13,14}$ However, this model is challenged by several independent observations.

Firstly, Btk can also act as adaptor molecule, independent of its catalytic activity. Expression of kinase-inactive Btk mutants have been shown to partially or fully reconstitute BCR-induced calcium mobilization in Btk-deficient chicken DT40 and human A20 mature B-cell lines. ${ }^{8,15,16}$ In addition, phosphorylation of PLC $\gamma 2$ upon BCR stimulation is apparently unaffected in human Btkdeficient B-cell lines. ${ }^{17}$ We have recently shown that during in vivo B-cell development, Btk function is in part independent of its catalytic activity, as transgenic expression of the kinase-inactive K430R-Btk mutant partially restored pre-B cell and B-cell defects in Btk-deficient mice. ${ }^{18}$

Secondly, in mature A20 B cells, Btk is essential for the recruitment of phosphatidylinositol phosphate 5-kinase (PIP5K) to the membrane, whereby enzymatic activity of Btk is not required for its association with PIP5K. ${ }^{15}$ Activation of PIP5K leads to local synthesis of PIP2, which is a common substrate shared by both PI3K and PLC $\gamma 2$. As a result, a positive feedback loop is initiated that allows Btk to stimulate the production (by PI3K) of PIP3, which is required for sustained Btk localization to the plasma membrane. At the same time, the shuttling function of Btk also provides substrate for sustained PLC $\gamma 2$ activity. ${ }^{12,15}$

Thirdly, Btk/SLP-65 double-deficient mice show an almost complete block in B-cell development at the pre-B-cell stage when 
compared with the partial block at this stage in SLP-65 or Btk single-mutant mice, ${ }^{19-21}$ indicating that Btk and SLP-65 partially function in parallel pathways. Both Btk and SLP-65 are essential for the regulation of pre-B-cell development, in particular by limiting pre-B-cell expansion at the transition of large cycling into small pre-B cells. ${ }^{22-26}$ SLP-65 ${ }^{-}$mice spontaneously develop pre-Bcell lymphomas expressing high levels of the pre-BCR on the cell surface. ${ }^{20,27}$ Although Btk-deficient mice do not develop pre-B-cell tumors, we recently found that Btk cooperates with SLP-65 as a tumor suppressor because the incidence of pre-B-cell lymphomas was significantly higher in Btk/SLP-65 double-mutant mice when compared with SLP-65 single-deficient mice. Moreover, transgenic expression of the constitutive active E41K-Y223F Btk mutant, which shows enhanced membrane localization, ${ }^{28,29}$ prevented tumor formation in Btk/SLP-65 double-deficient mice..$^{21}$

Importantly, the finding of defective SLP-65 expression in approximately $50 \%$ of childhood pre-B acute lymphoblastic leukemias (ALLs) indicated that SLP-65 also acts as a tumor suppressor in pre-B cells in humans. ${ }^{30}$ The loss of SLP-65 protein was apparently due to the incorporation of alternative exons into SLP-65 transcripts, leading to premature stop codons. ${ }^{30}$ Moreover, in human $\mathrm{Bcr}-\mathrm{Abl}^{+}$pre-B ALL, the activity of the Bcr-Abl1 kinase was found to be linked to defective pre-B signaling and the expression of the same aberrant SLP-65 transcripts. ${ }^{31}$ It was also observed that a large fraction of childhood pre-B ALL cases manifested aberrant Btk transcripts predicted to encode Btk proteins with a substantial kinase domain deletion. ${ }^{32}$

The mechanism by which Btk exerts its tumor suppressor function in pre-B cells independent of SLP-65 is currently unknown. By analogy with the findings in mature A20 B cells, Btk may function in pre-B cells as an adaptor molecule to localize PIP5K to the plasma membrane. However, it remains possible that this PIP5K shuttling mechanism that allows Btk to stimulate PLC $\gamma 2$ is dependent on the presence of SLP-65 (eg, as a scaffold molecule to bring Btk or other tyrosine kinase proteins, such as Syk or Src-family kinases, in close proximity with PLC $\gamma 2$ ).

In this study, we investigated whether Btk tumor suppressor function is independent of its kinase activity and at the same time whether SLP-65 is required for adaptor function of Btk. To this end, we crossed transgenic mice expressing the kinase-inactive Btk mutant K430R onto a Btk/SLP-65 double-deficient background and found that kinase-inactive Btk was able to functionally replace wild-type Btk as a tumor suppressor in the absence of SLP-65.

\section{Materials and methods}

\section{Mice and genotyping}

Btk-deficient mice ${ }^{33}$ and SLP-65-deficient mice 22 were on the C57BL/6 and Balb/c background, respectively. Wild-type and targeted alleles were identified as described previously. ${ }^{21,26}$ Btk-K430R transgenic mice, in which B-cell-specific expression of human Btk is under the control of the CD19 promoter region, were on a mixed C57B1/6 $\times$ FvB background. The presence of the Btk-K430R transgene was evaluated by polymerase chain reaction $(\mathrm{PCR}){ }^{18}$

\section{Flow cytometry and Ig ELISA}

Standard and intracellular flow cytometry and conjugated monoclonal antibodies (BD Biosciences, Mountain View, CA) have been described previously. ${ }^{26,33}$ The anti-SLC hybridoma LM34 ${ }^{34}$ was kindly provided by A. Rolink (University of Basel, Basel, Switzerland). Levels of Ig subclasses in serum were measured by sandwich enzyme-linked immunosorbent assay (ELISA). ${ }^{35}$

\section{Cell culture and Western blotting}

Total bone marrow (BM) cells or pre-B-lymphoma cells were cultured in the presence of $100 \mathrm{U} / \mathrm{mL}$ interleukin 7 (IL-7; Sigma-Aldrich, St Louis, MO) as described. ${ }^{26}$ For analysis of Btk phosphorylation, cells were stimulated with $10 \mu \mathrm{g} / \mathrm{mL} \mathrm{F}\left(\mathrm{ab}^{\prime}\right)_{2}$ fragment of polyclonal goat antimouse IgM (Jackson Immuno Research, Westgrove, PA) in RPMI 1640 at $37^{\circ} \mathrm{C}$. Total cell lysates of unstimulated and stimulated cells were immunoprecipitated overnight at $4^{\circ} \mathrm{C}$ with antiphosphotyrosine (pTyr-100; Cell Signaling Technology, Beverly, MA) and samples were blotted using standard sodium dodecyl sulfate-polyacrylamide gel electrophoresis (SDS-PAGE) procedures. ${ }^{36}$ Western blots were stained with anti-Btk C-20 (SantaCruz Biotechnology, Santa Cruz, CA) to detect Btk protein. Routinely, a small fraction of the total cell lysates were blotted with pTyr-100 to verify pre-BCR stimulation. $^{21}$

\section{$\mathrm{Ca}^{2+}$ mobilization assay}

Cells were loaded with $6 \mu \mathrm{g} / \mathrm{mL}$ indo- 1 acetoxymethylester (indo-1 AM; Molecular Probes Europe, Leiden, The Netherlands) at $37^{\circ} \mathrm{C}$ for 45 minutes and subsequently stained with phycoerythrin (PE)-labeled anti-CD4, anti-NK1.1, anti-Ter119, anti-CD11b, and fluorescein isothiocyanate (FITC)labeled anti-CD8 monoclonal antibodies (BD Biosciences). Cells were stimulated by $20 \mu \mathrm{g} / \mathrm{mL} \mathrm{F}\left(\mathrm{ab}^{\prime}\right)_{2}$ fragment of polyclonal goat antimouse $\operatorname{IgM}$ (Jackson Immuno Research) and subsequently by $2 \mu \mathrm{g} / \mathrm{mL}$ ionomycin as a positive control. Fluorescence ratios (FL-5/FL-4) were measured on a FACS-VantageSE/DiVa (BD Biosciences) and data for $\mathrm{B}$ cells $\left(\mathrm{CD} 4^{-} \mathrm{CD} 8 \mathrm{NK} 1.1^{-}\right.$Ter119 ${ }^{-} \mathrm{CD}_{11 b^{-}}$) were analyzed by FlowJo (Tree Star, Ashland, OR) multiparameter flow application.

\section{Results}

\section{Kinase-inactive Btk stimulates calcium mobilization in mature $B$ cells}

We previously showed that transgenic expression of the kinasedead Btk mutant K430R partially rescued Btk function in vivo, including the induction of nuclear factor $\kappa \mathrm{B}(\mathrm{NF}-\kappa \mathrm{B})$ and the expression of $\mathrm{Bcl}-\mathrm{x}_{\mathrm{L}}$ and cyclin D2 in splenic $\mathrm{B}$ cells upon BCR stimulation. ${ }^{18}$ To investigate whether these effects are mediated by the ability of K430R-Btk to potentiate calcium flux, we evaluated calcium mobilization in splenic $\mathrm{B}$ cells in response to BCR engagement in K430R-Btk transgenic mice on a Btk-deficient background, in comparison with wild-type and Btk-deficient mice (Figure 1A). Btk-deficient B cells showed a significantly reduced BCR-mediated $\mathrm{Ca}^{2+}$ signal when compared with wild-type B cells. Transgenic K430R-Btk provided complete correction of the $\mathrm{Ca}^{2+}$ response in Btk-deficient B cells, indicating that Btk stimulates calcium flux independent of its catalytic activity.

\section{SLP-65 is not required for pre-BCR-induced tyrosine phosphorylation}

If Btk and SLP-65 can function independently in parallel pathways in pre-B cells, this implies that Btk can be activated in the absence of SLP-65. It was previously shown that in SLP- $65^{-}$pre-B-cell lines, established by long-term IL-7-dependent bone marrow culture, the pre-BCR is signaling competent, whereby pre-BCR stimulation led to phosphorylation of Ig- $\alpha$, Syk, Lyn, PI3K, and PLC $\gamma 2 .{ }^{20}$ We evaluated phosphorylation of Btk in stable SLP-65deficient pre-B-cell lines expressing high levels of pre-BCR on the cell surface, which we established from SLP-65 ${ }^{-}$pre-B-lymphoma cells. ${ }^{21}$ Pre-B cells were stimulated with antibodies specific for Ig $\mu$ $\mathrm{H}$ chain, tyrosine-phosphorylated proteins were immunoprecipitated from total cellular lysates using pTyr-specific monoclonal 


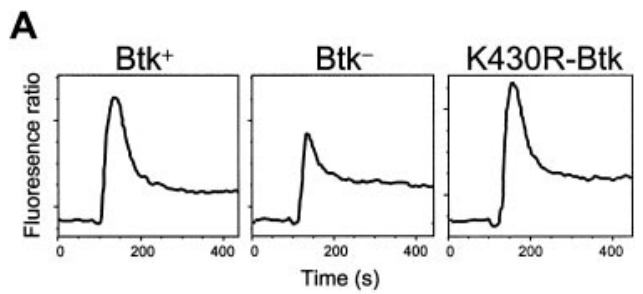

B

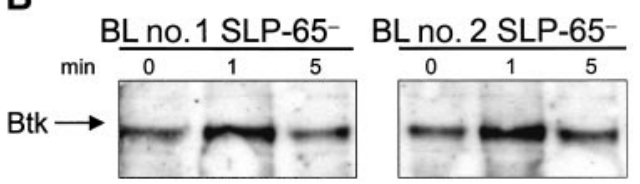

Figure 1. Kinase domain-independent and SLP-65-independent function of Btk. (A) K430R-Btk stimulates calcium flux in response to BCR activation. Indo-1 AM-loaded splenic $B$ cells of the indicated mice were stimulated with anti-lgM $F\left(a b^{\prime}\right)_{2}$ fragments and calcium flux was monitored. The plots are representative for 2 to 4 mice of each genotype. (B) Btk phosphorylation in the absence of SLP-65. Cultured cells from 2 different SLP-65- ${ }^{-}$pre-B-cell lymphomas (BL no. 1 and BL no. 2) were either not stimulated or stimulated for 1 or 5 minutes with polyclonal anti-IgM $F\left(a b^{\prime}\right)_{2}$ fragments. The presence of Btk in antiphosphotyrosine immunoprecipitates from total cellular lysates was analyzed by Western blotting using Btk-specific antibodies.

antibodies, and Btk protein was identified by blotting with a Btk-specific antibody. As shown in Figure 1B, pre-BCR stimulation in SLP-65-deficient pre-B cells resulted in Btk phosphorylation. Similar results were obtained in pre-BCR stimulation of short-term IL-7-dependent bone marrow-derived cultured pre-B cells from tumor-free SLP-65- mice (data not shown). These findings show that in murine pre-B cells, Btk can be phosphorylated upon pre-BCR stimulation in the absence of SLP-65.

\section{Transgenic K430R-Btk expression partially corrects the defects in B-cell development in Btk/SLP-65 double-mutant mice}

Mice deficient for either Btk or SLP-65 have a partial block in $\mathrm{B}$-cell development at the pre-B-cell stage in the BM, whereas Btk/SLP-65 double-deficient mice have an almost complete block at this stage. ${ }^{19,22-26}$ We have previously shown that expression of transgenic kinase-inactive K430R-Btk is able to partially rescue the mild differentiation defects at the pre-B-cell stage and the more severe block in peripheral B-cell maturation in Btk-deficient mice. ${ }^{18}$ To investigate to what extent the K430R-Btk transgene can reconstitute the almost complete pre-B-cell differentiation block in Btk/SLP-65 double-deficient mice, we compared the size of the B-cell subpopulations in spleen and BM by flow cytometry in 6 different mouse groups: $\mathrm{Btk}^{+}, \mathrm{Btk}^{-}$, or K430R-Btk transgenic on a $\mathrm{Btk}^{-}$background, each of which were either SLP- $65^{+}$or SLP- $65^{-}$.
A
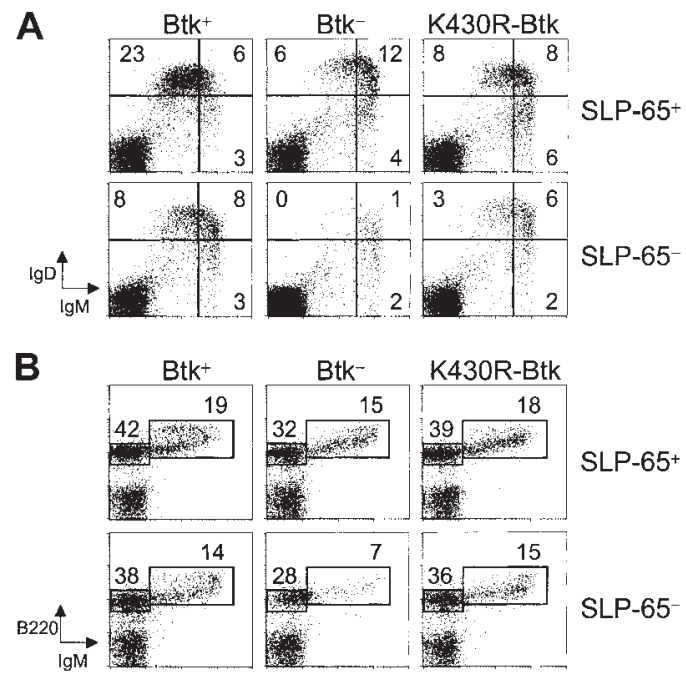

C

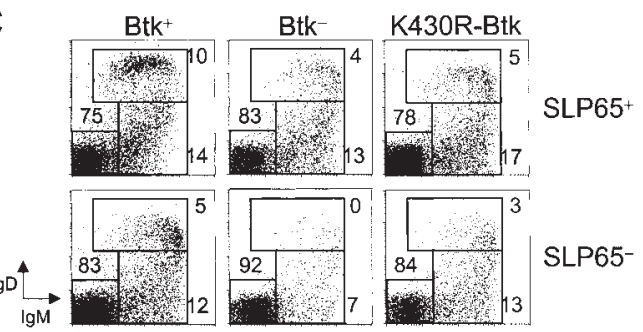

Figure 2. K430R-Btk partially corrects the defects in B-cell development in Btk/SLP-65 double-mutant mice. Flow cytometric analysis of $(A)$ spleen and $(B)$ bone marrow of $\mathrm{Btk}^{+}, \mathrm{Btk}^{-}$, and K430R-Btk mice on either the SLP-65 ${ }^{+}$or the SLP-65background. (A) lgM/lgD profiles of total splenic lymphoid cells, (B) lgM/B220, and (C) $\mathrm{IgM} / \mathrm{lgD}$ profiles of total bone marrow lymphoid cells. Lymphoid cells were electronically gated on the basis of forward and side scatter characteristics. Data are presented as dot plots and the percentages within the indicated quadrants or gates are given. The plots are representative for 5 to 14 mice of each genotype.

In agreement with our reported findings, ${ }^{18}$ reconstitution with K430R-Btk partially overcame the block in peripheral B-cell maturation present in Btk-deficient mice (Table 1; Figure 2A). Also, consistent with previous findings, ${ }^{19,21}$ the size of the total splenic B-cell population in Btk/SLP-65 double-deficient mice was severely reduced $\left(\sim 1.7 \times 10^{6}\right.$ cells $)$ when compared with wildtype or each of the single-deficient mice (Table 1). Expression of K430R-Btk in Btk/SLP-65 double-deficient mice increased the size of the total B-cell population in the spleen with a factor of approximately 4 (to $7.1 \times 10^{6}$ cells). This reconstitution was only partial, since the total numbers of splenic B cells in SLP-65 ${ }^{-}$mice were significantly higher $\left(\sim 22 \times 10^{6}\right.$ cells; Table 1$)$. Nevertheless,

Table 1. Absolute numbers of B-cell subpopulations in spleen and bone marrow

\begin{tabular}{|c|c|c|c|c|c|c|}
\hline & \multirow[b]{2}{*}{$\mathrm{n}^{*}$} & \multirow[b]{2}{*}{ Spleen† } & \multicolumn{4}{|c|}{ Bone marrow } \\
\hline & & & Pro-B cells $†$ & Pre-B cells $†$ & Immature $B$ cells $\dagger$ & Mature B cells $\dagger$ \\
\hline Mouse strain & NA & $\mathrm{B}^{2} 20^{+}$ & $\mathrm{B} 220^{+} \mathrm{C}^{-}$ & $\mathrm{B} 220^{+} \mathrm{C} \mu^{+} \operatorname{lgM}^{-}$ & $\lg ^{+} \lg D^{-}$ & $\lg ^{+} \lg \mathrm{D}^{+}$ \\
\hline $\mathrm{Btk}^{+}$ & $14(5)$ & $61.0 \pm 9.3$ & $2.6 \pm 0.3$ & $13.8 \pm 1.1$ & $3.9 \pm 1.3$ & $3.7 \pm 0.7$ \\
\hline $\mathrm{Btk}^{-}$ & $13(5)$ & $11.9 \pm 1.3$ & $3.0 \pm 0.3$ & $11.9 \pm 1.0$ & $3.9 \pm 0.3$ & $2.0 \pm 0.2$ \\
\hline K430R-Btk & 5 & $23.1 \pm 4.5$ & $2.8 \pm 0.4$ & $12.8 \pm 2.0$ & $3.8 \pm 0.8$ & $2.0 \pm 0.7$ \\
\hline SLP-65- & 5 & $21.7 \pm 5.0$ & $3.0 \pm 0.5$ & $12.8 \pm 2.3$ & $3.1 \pm 0.6$ & $1.5 \pm 0.4$ \\
\hline Btk $^{-}$SLP-65- & 5 & $1.7 \pm 0.3$ & $3.7 \pm 0.3$ & $8.2 \pm 1.6$ & $1.1 \pm 0.3$ & $0.1 \pm 0.0$ \\
\hline K430R-Btk/SLP-65- & 7 & $7.1 \pm 1.6 \ddagger$ & $2.8 \pm 0.3$ & $10.4 \pm 1.6$ & $2.6 \pm 0.4$ & $0.7 \pm 0.2$ \\
\hline
\end{tabular}

NA indicates not applicable.

${ }^{\star}$ Number of mice analyzed; the numbers in parentheses indicate that the B-cell population in spleen was analyzed in 5 mice per group.

†Absolute number as average $\pm \operatorname{SEM}\left(\times 10^{6}\right)$.

$\ddagger$ The absolute numbers of splenic B220 ${ }^{+}$cells in K430R-Btk/SLP-65- mice were significantly different from the values in SLP-65 $5^{-}$mice $(P=.008)$ and in Btk/SLP-65 double-deficient mice $(P=.025)$. 
the residual $\mathrm{B}$ cells present, which failed to differentiate into IgM $^{\text {low }} \operatorname{IgD}{ }^{\text {high }} \mathrm{B}$ cells in Btk/SLP-65 double-deficient mice, in part differentiated to $\operatorname{IgM}^{\text {low }} \operatorname{IgD}^{\text {high }}$ mature B cells when the K430R-Btk transgene was present (Figure 2A).

In the bone marrow, the $\mathrm{Btk}^{-}, \mathrm{K} 430 \mathrm{R}-\mathrm{Btk}$, and SLP- $65^{-}$mice differed from wild-type mice by an approximately $50 \%$ reduction in the size of the mature $\operatorname{IgM}^{+} \operatorname{IgD}^{+}$population of recirculating $\mathrm{B}$ cells (Table 1). By contrast, the Btk/SLP-65 double-deficient mice manifested a dramatic reduction in the total numbers of both immature $\operatorname{IgM}^{+} \operatorname{IgD}^{-}$and mature $\operatorname{IgM}^{+} \operatorname{IgD}^{+} \mathrm{B}$ cells. Importantly, the size of the immature B-cell population was similar in K430RBtk/SLP-65 ${ }^{-}$mice $\left(\sim 2.6 \times 10^{6}\right)$ and SLP-65 ${ }^{-}$mice $\left(\sim 3.1 \times 10^{6}\right)$. Thus, in the absence of SLP-65, kinase-inactive K430R-Btk could functionally replace Btk at the developmental progression from pre-B cells into immature B cells. From the analysis of $\operatorname{IgM} / \operatorname{IgD}$ profiles of the $\mathrm{B}_{2} 20^{+}$population (Figure $2 \mathrm{C}$ ), it is clear that SLP-65 ${ }^{-}$and K430R-Btk/SLP-65 ${ }^{-}$mice contained similar fractions of $\mathrm{IgM}^{+} \mathrm{IgD}^{-}$immature $\mathrm{B}$ cells $(\sim 12 \%)$. Expression of the K430R-Btk transgene only partly restored the reduction of the size of the mature $\operatorname{IgM}^{+} \operatorname{IgD}^{+} \mathrm{B}$-cell population observed in Btk/SLP$65^{-}$mice (Figure 2B-C; Table 1 ).

In summary, these flow cytometric analyses show that expression of kinase-inactive Btk can partially correct the severe B-cell differentiation defect in Btk/SLP-65 double-deficient mice, whereby the level of reconstitution is more complete in immature B cells than in mature B cells. Therefore, these results indicate that in SLP-65-independent signaling pathways, Btk kinase activity is not critical for the transition of pre-B cells into immature B cells and is apparently more important for the maturation of peripheral $\mathrm{B}$ cells.

\section{Btk kinase activity is specifically required for IgG3 production}

To further investigate if the Btk kinase domain is essential for the synergistic role of Btk and SLP-65 in mature B cells, we investigated the serum Ig levels in the 6 mouse groups. When compared with wild-type mice, $\mathrm{Btk}{ }^{-}$and SLP- $65^{-}$mice have severely decreased levels of $\operatorname{IgM}$ and $\operatorname{IgG} 3$, whereas other isotypes are generally not affected. ${ }^{22-25,37}$ We have previously found that kinase-inactive Btk could significantly restore the decreased levels of IgM in serum of Btk-deficient mice, but only a limited correction of serum IgG3 was observed. ${ }^{18}$ As shown in Figure 3, Btk/SLP-65 double-deficient mice have reduced serum levels of all isotypes when compared with each of the singlemutant littermates. Serum Ig levels in K430R transgenic Btk/

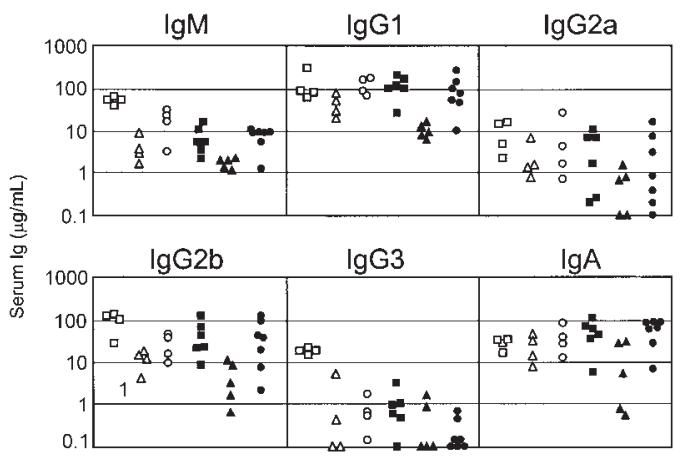

Figure 3. K430R-Btk restores serum Ig levels in Btk/SLP-65 double-mutant mice. Serum concentrations of $\lg M$, IgG1, $\lg G 2 a$, $\lg G 2 b, \lg G 3$, and $\lg A$, as determined by ELISA, are shown for the indicated mouse strains. Mice were 2 months of age and each symbol represents an individual animal. $\square$ indicates Btk ${ }^{+} ; \triangle$,

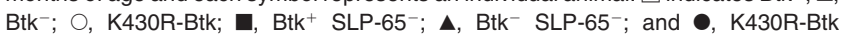
SLP-65
SLP-65 double-deficient mice were restored to levels similar to those found in SLP-65 single-deficient mice (Figure 3), except for IgG3. In fact, serum IgG3 levels in Btk/SLP-65 doubledeficient and K430R-Btk/SLP-65 ${ }^{-}$mice were very low, close to the lower detection limit of the ELISA.

Taken together, these results indicate that, with respect to late B-cell and plasma cell differentiation, in the absence of SLP-65, Btk mainly acts as a scaffolding protein. Only for the differentiation of B cells into IgG3-producing plasma cells is Btk kinase activity essential.

\section{K430R-Btk can functionally replace wild-type Btk in cellular maturation of SLP-65-deficient pre-B cells}

We have previously shown that Btk and SLP-65 are required for efficient transition of large cycling into small resting cytoplasmic $\mu^{+}$pre-B cells. ${ }^{21,26}$ In Btk or SLP-65 mutant mice, the down-regulation of the pro-B/large pre-B-cell-specific expression of SLC, CD43, and BP-1/6C3 aminopeptidase A and the up-regulation of $\mathrm{CD} 2, \mathrm{CD} 25$, and major histocompatibility complex (MHC) class II on small pre-B cells are impaired. A synergistic role for Btk and SLP-65 in this context is clear from the more pronounced defects in the modulation of these markers in Btk/SLP-65 double-deficient mice. ${ }^{21,26} \mathrm{We}$ also reported that kinase-inactive Btk is able to partially restore the defects at the pre-B-cell stage in Btk-deficient mice. ${ }^{18}$

To investigate the effects of K430R-Btk on pre-B-cell maturation in the absence of SLP-65, we compared the surface $\operatorname{IgM}^{-}$ pro-B/pre-B-cell fraction of $\mathrm{Btk}^{+}, \mathrm{Btk}^{-}$, and K430R-Btk transgenic mice, either on an SLP-65 ${ }^{+}$or an SLP- $65^{-}$background. We analyzed the expression of various developmentally regulated markers, including SLC, CD25, and MHC class II by flow cytometric analysis. In wild-type cells, only a small proportion of $\mu^{+}$pre-B cells expressed SLC ( $\sim 7 \%$; Figure $\left.4 \mathrm{~A}\right)$. As previously reported, ${ }^{18,21}$ transgenic $\mathrm{K} 430 \mathrm{R}-\mathrm{Btk}$ was able to restore the impaired down-regulation of SLC expression in Btk-deficient mice, whereas Btk/SLP-65 double-mutant mice manifested a much more severe defect in SLC down-regulation $\left(\sim 71 \% \mathrm{SLC}^{+}\right.$pre-B cells $)$. Interestingly, the levels of SLC expression in K430R-Btk/SLP-65 ${ }^{-}$ and SLP-65 ${ }^{-}$single-deficient pre-B cells were similar $(\sim 43 \%$ $\mathrm{SLC}^{+}$pre-B cells; Figure 4A), indicating that K430R-Btk could completely replace wild-type Btk function with respect to SLC down-regulation. Likewise, the levels of CD25 and MHC class II induction in K430R-Btk/SLP-65 ${ }^{-}$pre-B cells $(\sim 38 \%$ and $\sim 32 \%$, respectively) were comparable to those in SLP- $65^{-}$pre-B cells ( $\sim 56 \%$ and $\sim 21 \%$ ) and significantly higher than those in Btk/ SLP-65 double-mutant mice $(\sim 18 \%$ and $\sim 6 \%)$.

In summary, these analyses show that transgenic expression of kinase-inactive Btk is able to reduce the severe pre-B-cell maturation defects in Btk/SLP-65 double-deficient mice to a level that is comparable with SLP-65 single-deficient pre-B cells. Therefore, we conclude that in the absence of SLP-65, Btk signaling contributes to cellular maturation of pre-B cells, independent of its kinase activity.

\section{K430R-Btk can functionally replace wild-type Btk as a tumor suppressor in SLP-65- mice}

Concomitant deficiency of Btk strongly increases the frequency of pre-B-cell lymphoma formation in SLP-65- mice, demonstrating that Btk cooperates with SLP-65 as a tumor suppressor in pre-B cells. ${ }^{21}$ To investigate whether the tumor suppressor function of Btk is dependent on its kinase activity, we examined the capacity of 


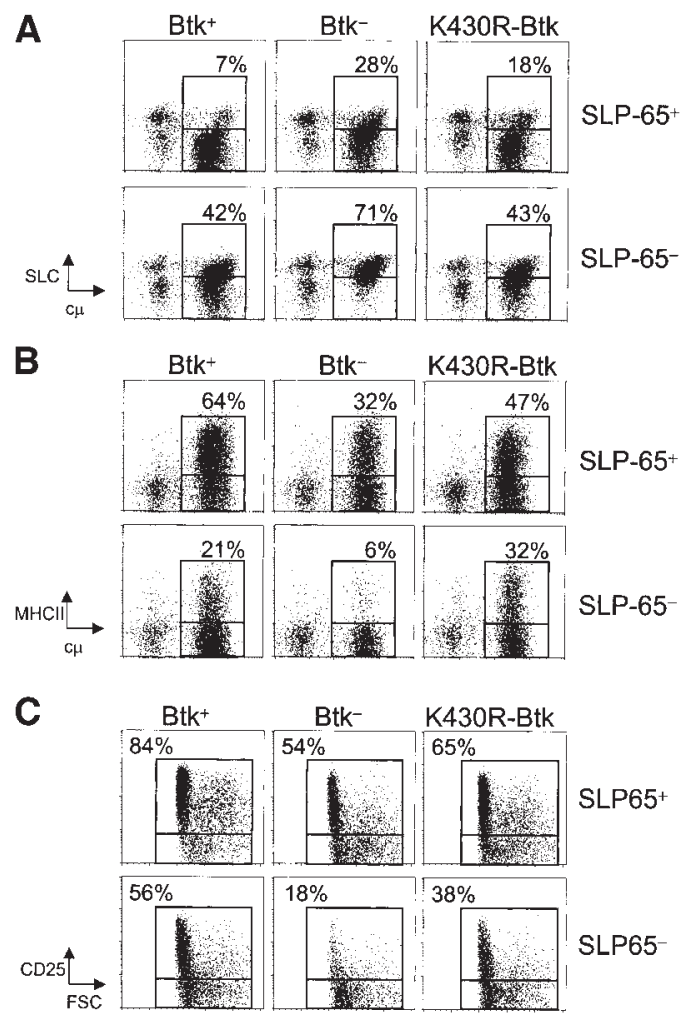

Figure 4. K430R-Btk corrects pre-B-cell maturation in Btk/SLP-65 doublemutant mice. Flow cytometric analysis of gated surface IgM-negative $\mathrm{B} 22 \mathrm{O}^{+}$ pro-/pre-B cells of $\mathrm{Btk}^{+}, \mathrm{Btk}^{-}$, and K430R-Btk mice on either an SLP-65 $5^{+}$or the SLP-65- background. $\mathrm{B}^{-} 20^{+} \mathrm{IgM}^{-}$cells were gated and analyzed for the expression of (A) cytoplasmic $\lg \mu \mathrm{H}$ chain $(\mathrm{C} \mu) / \mathrm{SLC},(\mathrm{B}) \mathrm{c} \mu / \mathrm{MHC}$, class II, and (C) forward scatter (FSC)/CD25. Data are displayed as dot plots and the percentage of cells within the indicated gates are given. Data shown are representative of 5 to 14 mice analyzed within each group.

K430R-Btk to substitute for Btk by following a panel of K430RBtk transgenic mice for 180 days. At this age, $18(\sim 15 \%)$ of 123 SLP-65 single-deficient mice developed a pre-B-cell lymphoma, whereas the Btk/SLP-65 double-deficient mice showed a frequency of approximately $44 \%$ (47 of 107). In contrast, only $7(\sim 14 \%)$ of 51 K430R-Btk/SLP-65-deficient mice developed a pre-B-cell lymphoma (Figure 5). From these results we conclude that transgenic kinase-inactive Btk can substitute for the endogenous wild-type Btk as a tumor suppressor in SLP-65-deficient mice, and therefore that the tumor suppressor function of Btk in pre-B cells is independent of its kinase activity.

\section{Discussion}

The finding that the block in B-cell differentiation and the associated pre-B-cell lymphoma formation in SLP-65 ${ }^{-}$mice is much more severe when Btk is concomitantly mutated suggested that the 2 proteins do not function only in a common signal transduction pathway. In this study, we demonstrate that during pre-B-cell development in the mouse, Btk functions as an adaptor molecule in an SLP-65-independent pathway.

We found that transgenic expression of the kinase-inactive K430R-Btk mutant was able to rescue the severe defects present in Btk/SLP-65 double-deficient pre-B cells to such an extent that the resulting phenotype was similar to that of SLP-65 single-deficient mice. In particular, we found that in the absence of SLP-65, Btk can act as an adaptor molecule that signals the modulation of SLC,
CD25, and MHC class II expression in pre-B cells. Importantly, the Btk kinase activity is also not required for its function as a tumor suppressor in SLP-65-deficient pre-B cells. Expression of the K430R-Btk transgene also restored the severe defects in mature B cells, although total B-cell numbers in the spleen and serum IgG3 levels were still reduced in K430R-Btk transgenic mice on the Btk/SLP-65 double-deficient background compared with SLP-65 single-deficient mice. This partial inability of K430R-Btk to substitute for Btk in mature B cells could reflect their absolute dependence on Btk kinase activity. Alternatively, this might be related to the expression pattern of the K430R-Btk transgene, which is under the control of the $C D 19$ promoter region. While the expression level is in the physiologic range in the bone marrow (1-2 $\times$ endogenous levels), this increases significantly as immature $\mathrm{B}$ cells differentiate to mature peripheral B cells $(\sim 15 \times$ overexpression). Therefore, it is possible that transgenic K430R-Btk expression partly has dominant-negative effects in vivo, which would be supported by our previous finding of reduced serum IgG3 levels and T-cell-independent IgM responses in K430R-Btk transgenic mice on a Btk wild-type background. ${ }^{18}$

The mechanism by which Btk functions as an adaptor molecule in pre-B cells is not known, but it is attractive to hypothesize that equivalent to the findings in mature A20 B cells, Btk may associate with PIP5K and recruit this enzyme to the plasma membrane. ${ }^{15}$ As overexpression of PIP5K was reported to lead to an increase in the sustained calcium response upon BCR activation, ${ }^{12}$ it is assumed that by providing the substrate PIP2 for PLC $\gamma 2$, PIP5K recruitment by Btk is sufficient to induce production of IP3 and DAG by PLC $y 2$. This mechanism would then explain the ability of kinaseinactive Btk to induce calcium mobilization in B cells upon BCR stimulation (Figure 1A). In this model, Btk should be recruited to the membrane and activated in an SLP-65-independent fashion. Although it has been proposed that Btk phosphorylation would be SLP-65 dependent, ${ }^{13}$ other data including ours favor a model in which Btk activation is SLP-65 independent (Fu et al ${ }^{38}$ and Chiu et $\mathrm{al}^{39}$; Figure 1B).

Taken together, our findings provide the biochemical basis for a parallel SLP-65-independent escape route when Btk is not able to phosphorylate PLC $\gamma 2$ directly: upon pre-BCR expression, Lyn, Syk, and PI3K are activated, resulting in phosphorylation and membrane translocation of Btk. Activated Btk is able to recruit PIP5K, which provides substrate for both PLC $\gamma 2$ and PI3K. This mechanism may also explain the phenotype of CD19/SLP-65 double-deficient mice, in which pre-B-cell differentiation was also almost completely blocked, ${ }^{27}$ quite similar to the phenotype of

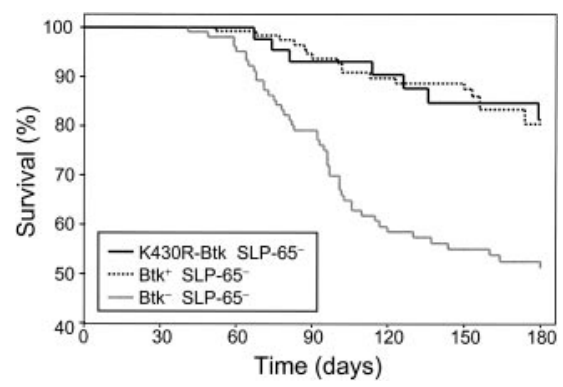

Figure 5. Tumor suppressor function of Btk is not dependent on its kinase activity. Kaplan-Meier tumor-free survival estimates for SLP-65- $(n=123)$, Btk- SLP$65^{-}(n=107)$, and K430R-Btk mice on the Btk ${ }^{-}$SLP- $65^{-}$background $(n=51)$. Tumor-free survival in Btk/SLP-65 double-deficient mice was significantly reduced compared with SLP-65 ${ }^{-}$mice $(P<.00001$, by log-rank) and to K430R-Btk/SLP-65 mice $(P<.0008)$. Tumor-free survival in the SLP-65- and K430R-Btk/SLP-65 ${ }^{-}$ groups of mice was not significantly different. 
Btk/SLP-65 double-deficient mice. ${ }^{19,21}$ From the findings in CD19/ SLP-65 double-deficient mice, it was concluded that the pre-B-cell transition, including cell cycle progression, down-regulation of recombination-activating gene 2 (RAG-2) protein, and SLC expression, as well as transcriptional activation of the Ig $\kappa$ locus, which is principally mediated by SLP-65, can also be (inefficiently) executed by CD19. ${ }^{27}$ In addition, in reconstituted myeloma cells, CD19 has been shown to be necessary for efficient BCR-mediated activation of $\mathrm{Btk},{ }^{40}$ most likely through the PI3K pathway and the production of PIP3 in concert with Lyn- or Syk-mediated phosphorylation of Btk. ${ }^{41,42}$ Therefore, we propose that the SLP-65independent parallel PLC $\gamma 2$ activation pathway in pre-B cells requires both Btk (as an adaptor protein) and CD19-dependent PIP3 synthesis by PI3K. In this model, a direct interaction between Btk and PLC $\gamma 2$ is not required and Btk and PIP5K would interact in the absence of SLP-65.

A possibility remains that SLP-65 deficiency can be compensated by the presence of redundant proteins because the concomitant absence of linker of activated T cells (LAT) in SLP-65- mice resulted in an almost complete block at the large pre-B-cell stage. ${ }^{43}$ Furthermore, it was shown that upon pre-BCR engagement, LAT recruits PLC $\gamma 2$ to the pre-BCR by association with Ig- $\alpha$ and the SLP-65 homologue SLP-76. These results indicate that LAT/ SLP-76 can rescue PLC $\gamma 2$ activation in the absence of SLP-65. ${ }^{43}$ However, in this model the capacity of LAT/SLP-76 to replace
SLP-65 function would be dependent on the presence of Btk as an adaptor molecule. Although SLP-65 or LAT/SLP-76, together with Btk, may serve as a scaffold to bring other kinase proteins in close proximity to PLC $\gamma 2$, phosphorylation of 2 of 4 regulatory tyrosines in PLC $\gamma 2$ appears to be entirely dependent upon Btk/Tec-family kinases, whereas Syk/zeta-associated protein 70 (ZAP-70) fails to modify these sites. ${ }^{44}$ Further experiments are required to investigate if Btk can interact with LAT or SLP-76.

In summary, our results show that Btk not only activates PLC $\gamma 2$ by phosphorylation in a complex with SLP-65 but also has a scaffolding function in an SLP-65-independent pathway in pre-B cells. This kinase-independent Btk function provides the molecular basis of the observed cooperation of Btk with SLP-65 as a tumor suppressor in pre-B cells. Based on recent findings in mature B cells, ${ }^{15}$ we hypothesize that Btk tumor suppressor function involves its capacity to recruit PIP5K to the membrane, which allows Btk to provide substrate for PLC $\gamma 2$.

\section{Acknowledgments}

We thank K. Dahlenborg, M. van Zelm, E. de Haas (Dept Immunology), and H. Diepstraten (Erasmus MC Animal Facility) for their assistance at various stages of the project.

\section{References}

1. Meffre E, Casellas R, Nussenzweig MC. Antibody regulation of B cell development. Nat Immunol. 2000;1:379-385.

2. Melchers F, ten Boekel E, Seidl T, et al. Repertoire selection by pre-B-cell receptors and B-cell receptors, and genetic control of B-cell development from immature to mature B cells. Immunol Rev. 2000;175:33-46.

3. Rolink AG, Winkler T, Melchers F, Andersson J. Precursor B cell receptor-dependent B cell proliferation and differentiation does not require the bone marrow or fetal liver environment. J Exp Med. 2000;191:23-32.

4. Ohnishi K, Melchers F. The nonimmunoglobulin portion of lambda5 mediates cell-autonomous pre-B cell receptor signaling. Nat Immunol. 2003; 4:849-856.

5. Guo B, Kato RM, Garcia-Lloret M, Wahl MI, Rawlings DJ. Engagement of the human pre-B cell receptor generates a lipid raft-dependent calcium signaling complex. Immunity. 2000;13:243-253.

6. Kouro T, Nagata K, Takaki S, et al. Bruton's tyrosine kinase is required for signaling the $C D 79 \mathrm{~b}$ mediated pro-B to pre-B cell transition. Int Immunol. 2001;13:485-493.

7. Schebesta M, Pfeffer PL, Busslinger M. Control of pre-BCR signaling by Pax5-dependent activation of the BLNK gene. Immunity. 2002;17:473-485.

8. Takata M, Kurosaki T. A role for Bruton's tyrosine kinase in B cell antigen receptor-mediated activation of phospholipase C-gamma 2. J Exp Med. 1996;184:31-40.

9. Watanabe D, Hashimoto S, Ishiai M, et al. Four tyrosine residues in phospholipase $\mathrm{C}$-gamma 2 , identified as Btk-dependent phosphorylation sites, are required for $B$ cell antigen receptorcoupled calcium signaling. J Biol Chem. 2001; 276:38595-38601.

10. Hendriks RW, Middendorp S. The pre-BCR checkpoint as a cell-autonomous proliferation switch. Trends Immunol. 2004;25:249-256.

11. Rawlings DJ, Scharenberg AM, Park H, et al. Activation of BTK by a phosphorylation mechanism initiated by SRC family kinases. Science. 1996; 271:822-825.
12. Scharenberg AM, El-Hillal O, Fruman DA, et al. Phosphatidylinositol-3,4,5-trisphosphate (Ptdlns3,4,5-P3)/Tec kinase-dependent calcium signaling pathway: a target for SHIP-mediated inhibitory signals. EMBO J. 1998;17:1961-1972.

13. Baba $Y$, Hashimoto $S$, Matsushita M, et al. BLNK mediates Syk-dependent Btk activation. Proc Nat Acad Sci U S A. 2001;98:2582-2586.

14. Kurosaki T, Tsukada S. BLNK: connecting Syk and Btk to calcium signals. Immunity. 2000;12: $1-5$.

15. Saito K, Tolias KF, Saci A, et al. BTK regulates Ptdlns-4,5-P(2) synthesis: importance for calcium signaling and PI3K activity. Immunity. 2003;19: 669-677.

16. Tomlinson MG, Woods DB, McMahon M, et al. A conditional form of Bruton's tyrosine kinase is sufficient to activate multiple downstream signaling pathways via PLC Gamma 2 in B cells. BMC Immunol. 2001;2:4.

17. Fluckiger AC, Li Z, Kato RM, et al. Btk/Tec kinases regulate sustained increases in intracellular $\mathrm{Ca} 2$ + following $\mathrm{B}$-cell receptor activation. EMBO J. 1998;17:1973-1985.

18. Middendorp S, Dingjan GM, Maas A, Dahlenborg $\mathrm{K}$, Hendriks RW. Function of Bruton's tyrosine kinase during $B$ cell development is partially independent of its catalytic activity. J Immunol. 2003; 171:5988-5996.

19. Jumaa $H$, Mitterer $M$, Reth $M$, Nielsen PJ. The absence of SLP65 and Btk blocks B cell development at the preB cell receptor-positive stage. Eur J Immunol. 2001;31:2164-2169.

20. Flemming $\mathrm{A}$, Brummer $\mathrm{T}$, Reth $\mathrm{M}$, Jumaa $\mathrm{H}$. The adaptor protein SLP-65 acts as a tumor suppressor that limits pre-B cell expansion. Nat Immunol. 2003;4:38-43.

21. Kersseboom R, Middendorp S, Dingjan GM, et al. Bruton's tyrosine kinase cooperates with the $\mathrm{B}$ cell linker protein SLP-65 as a tumor suppressor in pre-B cells. J Exp Med. 2003;198:91-98.

22. Jumaa $\mathrm{H}$, Wollscheid B, Mitterer M, Wienands J, Reth M, Nielsen PJ. Abnormal development and function of $B$ lymphocytes in mice deficient for the signaling adaptor protein SLP-65. Immunity. 1999;11:547-554.

23. Pappu R, Cheng AM, Li B, et al. Requirement for $B$ cell linker protein (BLNK) in B cell development. Science. 1999;286:1949-1954.

24. Hayashi K, Nittono R, Okamoto N, et al. The B cell-restricted adaptor BASH is required for normal development and antigen receptor-mediated activation of B cells. Proc Natl Acad Sci U S A. 2000;97:2755-2760.

25. Xu S, Tan JE, Wong EP, Manickam A, Ponniah S, Lam KP. B cell development and activation defects resulting in xid-like immunodeficiency in BLNK/SLP-65-deficient mice. Int Immunol. 2000; 12:397-404.

26. Middendorp S, Dingjan GM, Hendriks RW. Impaired precursor B cell differentiation in Bruton's tyrosine kinase-deficient mice. J Immunol. 2002; 168:2695-2703.

27. Hayashi K, Yamamoto M, Nojima T, Goitsuka R, Kitamura D. Distinct signaling requirements for Dmu selection, IgH allelic exclusion, pre-B cell transition, and tumor suppression in B cell progenitors. Immunity. 2003;18:825-836.

28. Li T, Tsukada S, Satterthwaite A, et al. Activation of Bruton's tyrosine kinase (BTK) by a point mutation in its pleckstrin homology $(\mathrm{PH})$ domain. Immunity. 1995;2:451-460.

29. Varnai P, Rother KI, Balla T. Phosphatidylinositol 3-kinase-dependent membrane association of the Bruton's tyrosine kinase pleckstrin homology domain visualized in single living cells. J Biol Chem. 1999;274:10983-10989.

30. Jumaa H, Bossaller L, Portugal K, et al. Deficiency of the adaptor SLP-65 in pre-B-cell acute lymphoblastic leukaemia. Nature. 2003;423:452456.

31. Klein F, Feldhahn N, Harder L, et al. The BCR ABL1 kinase bypasses selection for the expression of a pre-B cell receptor in pre-B acute lymphoblastic leukemia cells. J Exp Med. 2004;199: 673-685.

32. Goodman PA, Wood CM, Vassilev AO, Mao C, 
Uckun FM. Defective expression of Bruton's tyrosine kinase in acute lymphoblastic leukemia. Leuk Lymphoma. 2003;44:1011-1018.

33. Hendriks RW, de Bruijn MF, Maas A, Dingjan GM, Karis A, Grosveld F. Inactivation of Btk by insertion of lac Z reveals defects in B cell development only past the pre-B cell stage. EMBO J. 1996;15: 4862-4872.

34. Karasuyama H, Rolink A, Shinkai Y, Young F, Alt FW, Melchers F. The expression of Vpre-B/ lambda 5 surrogate light chain in early bone marrow precursor B cells of normal and B celldeficient mutant mice. Cell. 1994;77:133-143.

35. Dingjan GM, Maas A, Nawijn MC, et al. Severe B cell deficiency and disrupted splenic architecture in transgenic mice expressing the $\mathrm{E} 41 \mathrm{~K}$ mutated form of Bruton's tyrosine kinase. EMBO J. 1998; 17:5309-5320.
36. de Weers M, Brouns GS, Hinshelwood S, et al. $B$-cell antigen receptor stimulation activates the human Bruton's tyrosine kinase, which is deficient in X-linked agammaglobulinemia. J Biol Chem. 1994;269:23857-23860.

37. Khan WN, Alt FW, Gerstein RM, et al. Defective B cell development and function in Btk-deficient mice. Immunity. 1995;3:283-299.

38. Fu C, Turck CW, Kurosaki T, Chan AC. BLNK: a central linker protein in B cell activation. Immunity. 1998;9:93-103.

39. Chiu CW, Dalton M, Ishiai M, Kurosaki T, Chan AC. BLNK: molecular scaffolding through 'cis'mediated organization of signaling proteins. EMBO J. 2002;21:6461-6472.

40. Buhl AM, Cambier JC. Phosphorylation of CD19 Y484 and Y515, and linked activation of phosphatidylinositol 3-kinase, are required for B cell anti- gen receptor-mediated activation of Bruton's tyrosine kinase. J Immunol. 1999;162:4438-4446.

41. Li Z, Wahl MI, Eguinoa A, Stephens LR, Hawkins PT, Witte ON. Phosphatidylinositol 3-kinasegamma activates Bruton's tyrosine kinase in concert with Src family kinases. Proc Natl Acad Sci U S A. 1997;94:13820-13825.

42. Saito K, Scharenberg AM, Kinet JP. Interaction between the Btk PH domain and phosphatidylinositol-3,4,5-trisphosphate directly regulates Btk. J Biol Chem. 2001;276:16201-16206.

43. Su Y, Jumaa H. LAT links the pre-BCR to calcium signaling. Immunity. 2003;19:295-305.

44. Humphries LA, Dangelmaier C, Sommer K, et al. Tec kinases mediate sustained calcium influx via sitespecific tyrosine phosphorylation of the phospholipase Cgamma Src homology 2-Src homology 3 linker. J Biol Chem. 2004;279:37651-37661. 